\title{
Haptoglobin 2-2 genotype, patient and graft survival in renal transplant recipients
}

\begin{abstract}
Background. Cardiovascular disease is the leading cause of death in renal transplant recipients (RTRs). An association between haptoglobin genotype 2-2 and cardiovascular disease has been found in patients with diabetes mellitus and liver transplant recipients. To date, the role of haptoglobin genotype after renal transplantation has not been studied.

Methods. In this single centre retrospective cohort study of 1975 adult Norwegian RTRs, transplanted between 1999 and 2011, we estimated the risk of all-cause and cardiovascular mortality and overall and death censored graft loss for patients with haptoglobin genotype 2-2 compared with genotype $2-1$ or $1-1$, after adjustment for confounders and competing risks.

Results. We found no associations between haptoglobin genotype 2-2 and cardiovascular mortality (subdistributional hazard ratio [SHR] 1.08, 95\% confidence interval [CI] 0.78-1.49, $\mathrm{p}=0.63)$. We also failed to detect any association between haptoglobin 2-2 genotype and all-cause mortality, overall graft loss and death censored graft loss. Similar results were found in the subpopulation of diabetic RTRs.

Conclusion. In this large cohort of kidney transplant recipients, we could not demonstrate any association between haptoglobin 2-2 genotype and patient or graft survival after renal transplantation.
\end{abstract}




\section{INTRODUCTION}

Cardiovascular disease (CVD) contributes to one third of all premature deaths worldwide ${ }^{1}$, and is the major cause of death in renal transplant recipients (RTRs) ${ }^{2}$. Oxidative stress induces endothelial dysfunction and increases the risk of $\mathrm{CVD}^{2,3}$. Several pro- and anti-oxidative pathways act simultaneously, including pathways that make use of haemoglobin $(\mathrm{Hb})$ and its detoxifier haptoglobin $(\mathrm{Hp})$. When $\mathrm{Hb}$ is released from erythrocytes during intravascular hemolysis, $\mathrm{Hp}$ binds the free $\mathrm{Hb}$ and the $\mathrm{Hp}-\mathrm{Hb}$ complex is cleared from the circulation by the macrophage CD163 receptor ${ }^{4}$. Free $\mathrm{Hb}$ is highly toxic through release of free iron generating reactive oxygen species, initiating and enhancing atherosclerosis ${ }^{4}$. Due to genetic polymorphism; Hp 1-1, 2-1, and 2-2 genotype, Hp proteins are functionally different in different individuals ${ }^{4}$. Hp 2 proteins are not cleared from the circulation by the macrophage CD163 receptor as efficiently as Hp 1 proteins ${ }^{4}$. Consequently, individuals with the Hp 2-2 genotype are less protected against $\mathrm{Hb}$-induced oxidative damage than individuals expressing Hp 1-1 or 2-1 genotypes ${ }^{5}$. In addition to $\mathrm{Hp}-\mathrm{Hb}$ complex clearance by macrophages, renal proximal tubule cells can also clear the Hp$\mathrm{Hb}$ complex ${ }^{6}$. However, renal clearance of the $\mathrm{Hp}-\mathrm{Hb}$ complex may cause increased iron concentration in the proximal tubular cells, leading to tubular damage, collagen deposition and ultimately fibrosis. This mode of clearance mostly occurs in Hp 2-2 genotype individuals due to their lower macrophage CD163 receptor clearance ${ }^{4}$.

The most compelling evidence for a role of Hp genotype in cardiovascular health has been found in both human and animal studies of diabetes mellitus ${ }^{4,7}$. In transplant medicine, the role of Hp genotype has scarcely been studied. In liver transplant recipients, donor Hp 2-2 genotype was 
associated with reduced patient and graft survival ${ }^{8}$. To the best of our knowledge, no previous study has assessed associations between Hp genotype and patient and graft survival after renal transplantation.

The aim of the present study was to investigate the association between Hp 2-2 genotype and cardiovascular mortality, after adjustment for confounders and competing risk from other causes of death. In addition, we assessed associations between Hp 2-2 genotype and all-cause mortality, overall renal graft loss and death censored renal graft loss. 


\section{PATIENTS AND METHODS}

\section{Study participants, design, endpoints, data collection and procedures}

From a total of 2746 consecutive patients who received a renal transplant at Oslo University Hospital Rikshospitalet, Norway between $30^{\text {th }}$ of September 1999 and $13^{\text {th }}$ of October 2011 , data on Hp genotype were available in 1975 patients. Baseline differences between included and not included study participants has been described elsewhere ${ }^{9,10}$. In short, adult patients not included in the study were older than the study participants. When stratifying for age categories, study participants more often had a living donor. Otherwise there were no significant differences between the two groups. Informed consent was obtained from all patients. Selection of study patients, data quality control, handling of missing data, fitting of regression models, definition of outcomes and the standard immunosuppressive protocol in this cohort have previously been described in detail ${ }^{9}$. Clinical data were extracted from patient records. Endpoint data were provided by The Norwegian Renal Registry, which is based upon annual reports from all nephrology units and includes all patients on renal replacement therapy in Norway. Overall renal graft loss included both grafts lost due to recipient death and death censored graft loss (return to dialysis and renal re-transplantation). There were less than $1 \%$ missing data for included patients. The immunosuppressive therapy consisted of a combination of prednisolone, a cell proliferation inhibitor and a calcineurin inhibitor (cyclosporine A or tacrolimus), with some minor variations in what drugs have been used during the study period. Induction therapy with basiliximab was given to all patients transplanted in the year 2000 and from 2007 to 2011 . Acute rejections were treated with intravenous methylprednisolone followed by an increased dose of oral prednisolone which was tapered over the following two months. Steroid-resistant rejections were treated with anti-thymocyte globulin or anti-CD3 monoclonal antibodies. 
Hp genotypes were determined using high-performance liquid chromatography (HPLC) at Section of Molecular Diagnostics, Clinical Biochemistry, Aalborg University Hospital, Denmark. We used a modified HPLC method as previously described ${ }^{11,12}$. In brief, HPLC was performed using an UltiMate 3000 pump, an UltiMate 3000 autosampler, an UltiMate 3000 Variable Wavelength Detector (Dionex Denmark A/S, Hvidovre, Denmark) and a HPLC system manager (Chromeleon Client version 6.80, Dionex Corporation, Sunnyvale, CA, USA). For chromatographic separation, a YarraTM $3 \mu \mathrm{m}$ SEC-3000 column (Phenomenex ${ }^{\circledR}$, Værløse, Denmark) was used. To vials containing $30 \mu \mathrm{L}$ plasma, $5 \mu \mathrm{L}$ haemoglobin solution (dilution 1:10) and subsequently, $1255 \mu \mathrm{L}$ PBS solution were added as the mobile phase. The flow rate was $1 \mathrm{ml} / \mathrm{min}$. Detection of the haptoglobin-haemoglobin complex elution was monitored at wavelength $418 \mathrm{~nm}$. We used human reference plasma with known Hp phenotype (H1511 pooled plasma, H0138 Hp 1-1, and H9762 Hp 2-2. SIGMA, Sigma-Aldrich Denmark a/s, Denmark) for comparison. Hp phenotype was determined from the curve shape and the relative retention time of the chromatograms.

\section{Statistical analysis}

Chi-square test, independent t-test and Mann-Whitney U-test evaluated differences in patient characteristics at the time of transplantation between patients with Hp 2-2 genotype and Hp 2-1 or 1-1 genotype as appropriate (Table 1).

The two main statistical approaches in the present study were a proportional hazard regression model for the subdistribution of competing risks ${ }^{13,14}$ with either time to death censored graft loss or cardiovascular mortality as the outcome variable, and standard Cox regression with all-cause 
mortality or overall renal graft loss as the outcome variable. The observational time started at the time of transplantation. Surviving patients were censored at $1^{\text {st }}$ January 2015. In addition to Hp genotype, the following pre-defined variables were included in the model: Recipient age, gender, donor age, current smoking, atherosclerotic disease (coronary artery disease, cerebrovascular disease and/or peripheral vascular disease), transplant era (year 1999 through 2006 versus year 2007 through 2011), first or previous renal transplantation, living or deceased donor, preemptive transplantation, time in dialysis therapy prior to transplantation, diabetes mellitus prior to transplantation or posttransplantation diabetes mellitus during the first year after transplantation, and type of calcineurin inhibitor (cyclosporine A or tacrolimus) used at ten weeks after transplantation. We also performed similar analyses in a subgroup consisting of patients that were diagnosed with post-transplantation diabetes mellitus during the first year after renal transplantation or had diabetes prior to transplantation $(n=470)$.

In addition, we assessed differences in biopsy proven acute rejection rates, as previously described $^{9}$, between Hp genotypes. For 156 patients transplanted during 2010, we had available data on histologically verified progression of fibrosis between six weeks and one year post$\operatorname{transplant}^{15}$. The proportion of progressors and non-progressors in each $\mathrm{Hp}$ genotype was compared. A two-sided $\mathrm{p}$-value $<0.05$ was considered statistically significant.

The study was approved by the Regional Committees for Medical and Health Research Ethics in Norway and was performed in accordance with the Declaration of Helsinki. 


\section{RESULTS}

Demographic and clinical characteristics of the study population are presented in Table 1 . No differences between the Hp 2-2 genotype group and the pooled Hp 2-1 and 1-1 genotype group were found.

During a median follow-up of 7.0 (interquartile range $=4.7-10.5$ ) years, there were 401 deaths, either due to cardiovascular disease $(n=156)$ or other causes $(n=245)$. A total of 568 renal grafts were lost, either due to recipient death $(n=335)$ or death censored graft loss $(n=233)$.

\section{Mortality}

After adjustment for potential confounders and competing risk from other causes of death, patients with Hp 2-2 genotype did not have an increased cardiovascular mortality risk compared with RTRs with Hp 1-1/2-1 genotype (subdistributional hazard ratio [SHR] 1.08, 95\% confidence interval $[\mathrm{CI}]$ 0.78-1.49, p=0.63) (Table 2). Lack of association between Hp 2-2 genotype and cardiovascular mortality was also found in a subgroup analysis of RTRs with diabetes prior to transplantation or post-transplantation diabetes (SHR 1.06, 95\% CI 0.58-1.95, $\mathrm{p}=0.84$ ). In addition, we did not find an association between Hp 2-2 genotype and all-cause mortality in the whole study population (hazard ratio [HR] $0.93,95 \%$ CI $0.79-1.10, \mathrm{p}=0.41$ ) or diabetic RTRs (HR 0.99, 95\% CI 0.69-1.44, $\mathrm{p}=0.98)$.

\section{Renal graft loss}

RTRs with Hp 2-2 genotype had no increased risk of overall graft loss (HR 0.92, 95\% CI 0.781.09, $\mathrm{p}=0.34$ ). Similarly, we found no association between Hp 2-2 genotype and death censored graft loss (SHR 0.95, 95\% CI 0.73-1.24, p=0.72). In RTRs with diabetes, there were no 
statistically significant association between Hp 2-2 genotype and overall graft loss (HR 0.76, 95\% CI 0.55-1.06, $\mathrm{p}=0.11$ ) or death censored graft loss (SHR 0.61, 95\% CI 0.34-1.07, $\mathrm{p}=0.09$ ). There were no differences in choice of immunosuppressive regimens between Hp genotypes (data not shown). Rates of biopsy proven acute rejection episodes were similar in $\mathrm{Hp} 2-2$ genotype as in Hp 1-1 and 2-1 genotypes both in early phase (first ten weeks after renal transplantation) and beyond the early post-transplant phase (data not shown). The proportion of patients with histologically verified progression of fibrosis during the first year post-transplant did not differ between Hp genotypes (data not shown). 


\section{DISCUSSION}

The present study found no association between Hp 2-2 genotype and cardiovascular mortality, all-cause mortality, overall renal graft loss or death censored graft loss. The distribution of Hp genotype was similar to previous studies (Hp 1-1: 13.2\%, Hp 2-1: 44.5\%, Hp 2-2: 42,3\%) ${ }^{16,17}$.

\section{Haptoglobin genotype and CVD}

Hp genotype is one of several factors that might induce oxidative mediated atherosclerosis, and its role in the development of CVD is not clearly established ${ }^{4}$. Hp 2-2 genotype is positively associated with myocardial infarction size ${ }^{18}$ and negatively associated with coronary bypass graft patency $^{19}$. However, most studies in non-diabetic patients have reported neutral ${ }^{4}$ or even beneficial associations with $\mathrm{Hp} 2-2$ genotype $^{20}$. In patients with diabetes, the evidence for an association between Hp 2-2 genotype and CVD is stronger. Macrophage clearance of the Hp-Hb complex is slower in $\mathrm{Hp} \mathrm{2-2}$ genotype individuals ${ }^{21}$, leaving more glycated $\mathrm{Hb}$ and reactive oxygen species in the circulation. A positive association between Hp 2-2 genotype and the risk of major cardiac events were found in the Strong Heart Study ${ }^{17}$ and The Munich Stent study ${ }^{16}$, and supplementation of the anti-oxidant vitamin $\mathrm{E}$ lowered the risk of cardiovascular events in diabetic patients with Hp 2-2 genotype in the ICARE study ${ }^{22}$.

In the present study, we found no association between Hp 2-2 genotype and cardiovascular mortality, even not in the subgroup of diabetic patients. It should be noted, however, that associations with mortality endpoints, being crude outcome variables, would not necessarily reflect possible associations between Hp 2-2 genotype and cardiovascular morbidity, including major cardiovascular events. Indeed, previous reports indicate a positive association between $\mathrm{Hp}$ 2-2 genotype and development of CVD, while a similar association with cardiovascular mortality 
has not been shown. Moreover, Hp 2-2 genotype is linked to atherosclerosis. There was a significant association between atherosclerotic disease prior to transplantation and cardiovascular mortality. In multivariable adjusted survival analysis, atherosclerotic disease prior to transplantation was introduced as a confounding variable, while it could also partly be an intermediate variable linking Hp 2-2 genotype to cardiovascular mortality. On the other hand, in univariate analyses, similar associations were found between Hp 2-2 genotype to cardiovascular mortality. Unfortunately, we did not have any data on cardiovascular events in this cohort.

\section{Haptoglobin genotype and kidney disease}

Previous reports indicate a positive association between $\mathrm{Hp}$ 2-2 genotype and decline in renal function and end-stage renal disease incidence in patients with diabetes ${ }^{23,24}$. Glomerular filtration of Hp 2-2 proteins increases iron deposition in and damage to renal proximal tubule cells ${ }^{6}$. In patients with end-stage renal disease, Hp 2-2 genotype was associated with higher high-sensitive C-reactive protein levels ${ }^{25}$, indicating a relationship between Hp genotype and inflammation. We found no association between Hp 2-2 genotype and overall or death censored graft loss. Although most of the Hp proteins are synthesized in the liver, where the recipient genotype determines the Hp protein product, there is some production of Hp proteins in the kidney, which for RTRs will be determined by the donor Hp genotype. Obviously, this may prelude any associations between recipient Hp genotype and renal graft survival.

\section{Haptoglobin genotype and organ transplantation}

In transplant medicine, only a few studies have investigated the role of Hp genotype. In liver transplant recipients, embracing 450 patients during 3 years follow-up, donor Hp 2-2 genotype compared with donor Hp 2-1 and 1-1 genotypes was associated with poorer patient and graft 
survival $^{8}$. Haptoglobin is predominantly synthesized by hepatocytes, hence donor Hp genotype determines which $\mathrm{Hp}$ protein will be produced in this patient group ${ }^{8}$. In heart transplant recipients, Hp 2-1 genotype, but not Hp 2-2 genotype, was associated with a higher risk of cardiac transplant vasculopathy ${ }^{26}$. Due to a small sample size this finding should be interpreted with caution and the biological rationale for an association between $\mathrm{Hp}$ 2-1 genotype and cardiac transplant vasculopathy remains unexplained. In RTRs, Hp 1-1 genotype compared with Hp 2-2 and 2-1 genotype was associated with increased risk for development of cutaneous squamous cell carcinoma $^{27}$. To date, the roles of either recipient or donor Hp genotype for most long-term outcomes after renal transplantation has not been investigated, including patient and graft survival.

\section{Strengths and limitations}

The major strength of the present study is the large cohort from a single center with only a few missing data. The present study also has several limitations, some of which has been addressed in previous sections. We have no information about donor $\mathrm{Hp}$ genotype. The study population almost exclusively consisted of Caucasian patients and the results may therefore not apply to other ethnical groups. Since haptoglobin genotyping was only performed in patients who attended a clinical visit ten weeks after renal transplantation, the survival analyses might have been influenced hampered by immortal time bias to some degree. The choice of mortality endpoints might have been to crude to detect an impact of Hp genotypes on cardiovascular health. Finally, we had no information on pre-existing cardiovascular disease. 


\section{CONCLUSION}

Recipient Haptoglobin genotype was not associated with either cardiovascular mortality, allcause mortality, overall and death censored graft loss after renal transplantation, including renal transplant recipients with diabetes mellitus. 


\section{REFERENCES}

1. Yusuf S, Reddy S, Ounpuu S, Anand S. Global burden of cardiovascular diseases: part I: general considerations, the epidemiologic transition, risk factors, and impact of urbanization. Circulation. 2001;104(22):2746-2753.

2. Israni AK, Snyder JJ, Skeans MA, et al. Predicting coronary heart disease after kidney transplantation: Patient Outcomes in Renal Transplantation (PORT) Study. American journal of transplantation : official journal of the American Society of Transplantation and the American Society of Transplant Surgeons. 2010;10(2):338-353.

3. Cai H, Harrison DG. Endothelial dysfunction in cardiovascular diseases: the role of oxidant stress. Circ Res. 2000;87(10):840-844.

4. Costacou T, Levy AP. Haptoglobin genotype and its role in diabetic cardiovascular disease. J Cardiovasc Transl Res. 2012;5(4):423-435.

5. Gueye PM, Glasser N, Ferard G, Lessinger JM. Influence of human haptoglobin polymorphism on oxidative stress induced by free hemoglobin on red blood cells. Clinical chemistry and laboratory medicine. 2006;44(5):542-547.

6. Levy AP, Asleh R, Blum S, et al. Haptoglobin: basic and clinical aspects. Antioxidants \& redox signaling. 2010;12(2):293-304.

7. Asaf R, Blum S, Roguin A, et al. Haptoglobin genotype is a determinant of survival and cardiac remodeling after myocardial infarction in diabetic mice. Cardiovascular diabetology. 2009;8:29.

8. Speeckaert R, Van Vlierberghe $H$, Troisi R, et al. Donor haptoglobin phenotype determines outcome following liver transplantation. Transplant international : official journal of the European Society for Organ Transplantation. 2011;24(6):619-626.

9. Eide IA, Jenssen T, Hartmann A, et al. The Association between Marine n-3 Polyunsaturated Fatty Acid Levels and Survival after Renal Transplantation. Clinical journal of the American Society of Nephrology : CJASN. 2015.

10. Eide IA, Halden TA, Hartmann A, Dahle DO, Asberg A, Jenssen T. Associations Between Posttransplantation Diabetes Mellitus and Renal Graft Survival. Transplantation. 2016.

11. Friis H, Gomo E, Nyazema N, et al. Iron, haptoglobin phenotype, and HIV-1 viral load: a cross-sectional study among pregnant Zimbabwean women. Journal of acquired immune deficiency syndromes. 2003;33(1):74-81.

12. Delanghe J, Allcock K, Langlois M, Claeys L, De Buyzere M. Fast determination of haptoglobin phenotype and calculation of hemoglobin binding capacity using high pressure gel permeation chromatography. Clin Chim Acta. 2000;291(1):43-51.

13. Singh SK, Kaplan B, Kim SJ. Multivariable Regression Models in Clinical Transplant Research: Principles and Pitfalls. Transplantation. 2015;99(12):2451-2457.

14. Noordzij M, Leffondre K, van Stralen KJ, Zoccali C, Dekker FW, Jager KJ. When do we need competing risks methods for survival analysis in nephrology? Nephrol Dial Transplant. 2013;28(11):2670-2677.

15. Dorje C, Reisaeter AV, Dahle DO, et al. Total inflammation in early protocol kidney graft biopsies does not predict progression of fibrosis at 1 year post-transplant. Clinical transplantation. 2016. 
16. Roguin A, Koch W, Kastrati A, Aronson D, Schomig A, Levy AP. Haptoglobin genotype is predictive of major adverse cardiac events in the 1-year period after percutaneous transluminal coronary angioplasty in individuals with diabetes. Diabetes care. 2003;26(9):2628-2631.

17. Levy AP, Hochberg I, Jablonski $K$, et al. Haptoglobin phenotype is an independent risk factor for cardiovascular disease in individuals with diabetes: The Strong Heart Study. Journal of the American College of Cardiology. 2002;40(11):1984-1990.

18. Chapelle JP, Albert A, Smeets JP, Heusghem C, Kulbertus HE. Effect of the haptoglobin phenotype on the size of a myocardial infarct. The New England journal of medicine. 1982;307(8):457-463.

19. Delanghe J, Cambier B, Langlois $M$, et al. Haptoglobin polymorphism, a genetic risk factor in coronary artery bypass surgery. Atherosclerosis. 1997;132(2):215-219.

20. De Bacquer D, De Backer G, Langlois M, Delanghe J, Kesteloot H, Kornitzer M. Haptoglobin polymorphism as a risk factor for coronary heart disease mortality. Atherosclerosis. 2001;157(1):161-166.

21. Viener HL, Levy AP. Haptoglobin genotype and the iron hypothesis of atherosclerosis. Atherosclerosis. 2011;216(1):17-18.

22. Milman U, Blum S, Shapira C, et al. Vitamin E supplementation reduces cardiovascular events in a subgroup of middle-aged individuals with both type 2 diabetes mellitus and the haptoglobin 2-2 genotype: a prospective double-blinded clinical trial.

Arteriosclerosis, thrombosis, and vascular biology. 2008;28(2):341-347.

23. Costacou T, Orchard TJ. The Haptoglobin genotype predicts cardio-renal mortality in type 1 diabetes. Journal of diabetes and its complications. 2015.

24. Orchard TJ, Sun W, Cleary PA, et al. Haptoglobin genotype and the rate of renal function decline in the diabetes control and complications trial/epidemiology of diabetes interventions and complications study. Diabetes. 2013;62(9):3218-3223.

25. Strandhave C, Svensson M, Krarup H, Christensen JH. Haptoglobin genotype and risk markers of cardiovascular disease in patients with chronic kidney disease. International journal of nephrology. 2013;2013:650847.

26. Densem CG, Wassel J, Brooks NH, Yonan N, Keevil B. Haptoglobin polymorphism influences the development of cardiac transplant vasculopathy. The Journal of heart and lung transplantation : the official publication of the International Society for Heart Transplantation. 2001;20(2):151.

27. Speeckaert R, Brochez L, Lambert J, et al. The haptoglobin phenotype influences the risk of cutaneous squamous cell carcinoma in kidney transplant patients. Journal of the European Academy of Dermatology and Venereology : JEADV. 2012;26(5):566-571. 